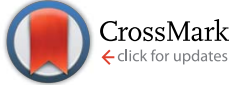

Cite this: RSC Adv., 2016, 6, 113025

Received 6th August 2016

Accepted 15th November 2016

DOI: 10.1039/c6ra19915a

www.rsc.org/advances

\section{Dual-functionalisation of gelatine nanoparticles with an anticancer platinum(II)-bisphosphonate complex and mineral-binding alendronate $\uparrow$}

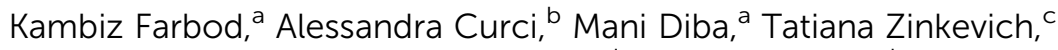 \\ Arno P. M. Kentgens, ${ }^{c}$ Michele lafisco, ${ }^{d}$ Nicola Margiotta $^{b}$ \\ and Sander C. G. Leeuwenburgh*a
}

In order to improve the efficacy of therapeutic systems to treat bone tumours, novel drug delivery vehicles should be developed that have strong and specific affinity to mineralised tissue and at the same time are able to release anticancer molecules locally in a controlled and sustained manner. Recently, we developed mineral-binding gelatine nanoparticles with enhanced affinity to calcium phosphate by conjugating alendronate (ALN) molecules onto their surface. Herein, we have enhanced the functionality of these nanoparticles by rendering them potentially therapeutically active via covalent linking of an anticancer platinum-bisphosphonate (Pt-BP) complex. Different functionalisation schemes and molar ratios between reactants were screened and the effective functionalisation of gelatine nanoparticles with Pt-BP (or with both Pt-BP and ALN) was assessed. Our results revealed that the degree of functionalisation could be tailored by varying the molar ratio of $\mathrm{Pt}-\mathrm{BP}$ and $\mathrm{ALN}$ relative to glutaraldehyde used as crosslinker. A sustained and tunable release of platinum as a function of the initial Pt-BP/ALN/ glutaraldehyde molar ratio was achieved for both Pt-BP- and dual-functionalised gelatine nanoparticles. Moreover, dual-functionalised gelatine nanoparticles also displayed a high affinity to hydroxyapatitecoated surfaces thanks to the presence of ALN. Summarising, it was demonstrated that mineral-binding gelatine nanoparticles can be loaded with tailored amounts of anticancer molecules, which may benefit the development of bone-seeking carriers for targeted delivery of drugs to treat bone tumours.

\section{Introduction}

Bone is a frequent site for metastases, which develop in up to $70 \%$ of patients with prostate cancer and breast cancer, ${ }^{\mathbf{1}}$ and in up to $30 \%$ of those with cancers of the lung, bladder and thyroid. ${ }^{2}$ Bone metastases are a health and economic burden as they are associated with numerous skeletal-related issues including pathological fractures, spinal cord compression, pain, need for radiation therapy or surgery to bone. ${ }^{3}$ Besides the devastating effect of cancer on bone tissue, current treatment modalities not specific to cancer cells result in damaging

${ }^{a}$ Department of Biomaterials, Radboud Institute for Molecular Life Sciences, Radboud University Medical Center, Philips van Leydenlaan 25, 6525 EX Nijmegen, The Netherlands.E-mail: sander.leeuwenburgh@radboudumc.nl

${ }^{b}$ Dipartimento di Chimica, Università degli Studi di Bari Aldo Moro, Via E. Orabona 4, 70125 Bari, Italy

${ }^{c}$ Department of Solid State NMR, Institute for Molecules and Materials, Radboud University, Heyendaalseweg 135, 6525 AJ Nijmegen, The Netherlands

${ }^{d}$ Institute of Science and Technology for Ceramics (ISTEC), National Research Council (CNR), Via Granarolo 64, 48018 Faenza, Italy

$\dagger$ Electronic supplementary information (ESI) available: Additional characterisation of functionalised gelatine nanoparticles and characterisation of hydroxyapatite sputter coating on titanium discs. See DOI: 10.1039/c6ra19915a normal cells and healthy tissues. ${ }^{4}$ In fact, a major disadvantage of anticancer drugs is their lack of selectivity for tumour tissue, which causes severe side effects and results in low cure rates. ${ }^{5}$ For instance, therapy with platinum-based chemotherapeutic molecules (e.g. cisplatin (cis-diamminedichloridoplatinum(II))), which are a group of key drugs used in the treatment of several types of cancers, are associated with various secondary effects including anemia, nausea, vomiting, neurotoxicity and nephrotoxicity. These side effects impair the patient's quality of life and can even be life-threatening. ${ }^{6-8}$

To overcome these side effects, the design of a new generation of molecules to target traditional anticancer platinum-based drugs to bone tissue is strongly required. ${ }^{8}$ To this end, platinumbisphosphonate complexes (i.e. bis-\{ethylenediamineplatinum(II)\}2-amino-1-hydroxyethane-1,1-diyl-bisphosphonate (Pt-BP) and bis\{ethylenediamineplatinum(II)\}-medronate) were recently developed. ${ }^{9-11}$ Bisphosphonate-functionalised platinum-based molecules not only target bone as a result of the bone-seeking properties of bisphosphonate moieties, ${ }^{\mathbf{1 2 - 1 4}}$ but also specifically select bone tumours ${ }^{\mathbf{1 5}}$ due to their higher metabolic activity as compared to healthy cells. ${ }^{\mathbf{1 6}}$

Traditional systemic delivery of chemotherapeutics, however, entails high systemic drug elimination through renal 
and urinary excretion and limited circulation time. ${ }^{17}$ On the contrary, chemotherapeutic drugs would be delivered more efficiently to bone tumours upon bone-specific and sustained delivery from suitable carriers, ${ }^{18,19}$ thereby reducing toxic side effects in unaffected tissues. To this end, novel and more effective drug delivery vehicles should be developed that have strong and specific affinity to mineralised tissue and are able to release anticancer drugs locally in a controlled and sustained manner. ${ }^{5,20}$

The intrinsic biocompatibility, biodegradability and ease of functionalisation of natural polymers are of great potential value for applications in tissue engineering, regenerative medicine, pharmaceutics and drug delivery. ${ }^{\mathbf{2 1 , 2 2}}$ Gelatine is a particularly attractive candidate material for drug delivery, since it is a low-cost proteinaceous biopolymer that has been extensively used for biomedical purposes thanks to its biocompatible, biodegradable and non-toxic properties. ${ }^{\mathbf{1 8 , 2 2 - 2 5}}$ Gelatine can be tuned with respect to its drug loading efficiency, ${ }^{26}$ degradation rate and release kinetics, ${ }^{25,27}$ which renders gelatine a remarkably versatile drug carrier for a wide variety of biomedical applications. ${ }^{27,28}$ For applications as a drug delivery vehicle, gelatine has been processed into different shapes and forms, such as hydrogels, ${ }^{29,30}$ sponges, ${ }^{31-34}$ coatings or films ${ }^{35-37}$ microparticles ${ }^{38-42}$ and nanoparticles. ${ }^{6,43-47}$ Gelatine microparticles have been used as carriers of anticancer drugs such as cisplatin. ${ }^{\mathbf{4 8 4 9}}$ Although cisplatin was only adsorbed onto gelatine microparticles, the anticancer effect was enhanced and prolonged as compared to carrier-free delivery of cisplatin alone. ${ }^{48}$ Microparticles, however, lack the mobility and size required for targeted delivery and are often entrapped in nontarget organs. ${ }^{38}$ Sub-micron or nanoparticles, on the contrary, have gained increased research interest, ${ }^{50}$ due to their significantly smaller size and thus increased specific surface area, which enhances the drug payload and facilitates targeted delivery to specific tissues. ${ }^{\mathbf{1}}$

Gelatine nanoparticles have been used for the delivery of both hydrophilic and hydrophobic anticancer drugs. ${ }^{18,52}$ Drug-loaded gelatine nanoparticles have also demonstrated rapid uptake and long-term retention in the tumour after administration..$^{\mathbf{1 8 , 1 9}}$ The superior efficacy of anticancer drug-loaded gelatine nanoparticles compared to free drug has been validated both in vitro (in cancer cell lines) and in vivo (in tumour-bearing animal models). ${ }^{18,19,52-55}$ Cytotoxicity analysis of anticancer drug-loaded gelatine nanoparticles against different cancer cell lines has shown higher anticancer activity as compared to the free drug. This has been attributed to greater endocytotic uptake of gelatine nanoparticles in cancer cells. ${ }^{\mathbf{5 2 , 5 6 , 5 7}}$ Gelatine nanoparticles, however, have not yet been developed for bone-specific drug delivery. Recently, we developed mineral-binding gelatine nanoparticles with enhanced affinity to calcium phosphate (CaP). ${ }^{12}$ Herein, we attempt to link-for the first time-novel bone-specific gelatine nanoparticles to a chemotherapeutic PtBP drug in order to render them potentially chemotherapeutically active. Pt-BP loading was done using two different methods (i.e. via covalent attachment or physical adsorption), while the effect of the drug loading was analysed in detail with respect to the physicochemical properties of the carriers including their mineral-binding capacity as well as the release kinetics of the drug. The functionalisation reactions were carried out using different reaction schemes and molar ratios between the reactants and were characterised using ${ }^{13} \mathrm{C}$ and ${ }^{31} \mathrm{P}$ Solid-State Nuclear Magnetic Resonance (SS-NMR) spectroscopy, EnergyDispersive X-ray (EDX) spectroscopy and Inductively Coupled Plasma-Optical Emission Spectrometry (ICP-OES). The efficacy of conjugation as well as the retention and release properties of the Pt-BP-loaded gelatine nanoparticles were investigated using ICP-OES. Finally, the influence of dual functionalisation with Pt$\mathrm{BP}$ and ALN on the affinity of gelatine nanoparticles to CaP was assessed using fluorescent microscopy.

\section{Experimental section}

\section{Materials}

Gelatine type B (from bovine skin, 225 Bloom, average molecular mass: $\sim 50 \mathrm{kDa}, \mathrm{pI} \sim 5, \sim 33$ millimoles of free amino groups $^{58}$ and 100-115 millimoles of carboxyl groups per $100 \mathrm{~g}$ gelatine) was purchased from Sigma-Aldrich. Glutaraldehyde (GA, 25 wt\% solution in water) was purchased from Acros Organics. Alendronic acid (ALN, (4-amino-1-hydroxybutylidene)diphosphonic acid) was purchased from AK Scientific, Inc. (AKSci, Union City, CA, USA). Acetone was purchased from Boom BV (Meppel, the Netherlands). 2-Ammonium-1hydroxyethane-1,1-diyl-bisphosphonic acid $\left(\mathrm{AHBP}^{-} \mathrm{H}_{5}\right)$ and $\left[\mathrm{Pt}\left(\mathrm{OSO}_{3}\right)\left(\mathrm{OH}_{2}\right)(\mathrm{en})\right]$ (en = ethylenediamine) were prepared following procedures reported previously. ${ }^{9}$ Milli-Q water was used to dissolve the platinum compounds. All other reagents were purchased from Sigma-Aldrich.

\section{Preparation and characterisation of Pt-BP complex}

$\left[\{\mathrm{Pt}(\mathrm{en})\}_{2}\left(\mu-\mathrm{AHBP}-\mathrm{H}_{2}\right)\right]\left(\mathrm{HSO}_{4}\right) \quad$ (bis-\{ethylenediamineplatinum(II) $\}$ 2-amino-1-hydroxyethane-1,1-diyl-bisphosphonate), Pt-BP (Fig. 1), was prepared according to already reported methods. ${ }^{16,59}$ Briefly, 2-ammonium-1-hydroxyethane-1,1-diyl-bisphosphonic acid $\left(\mathrm{AHBP}_{5} \mathrm{H}_{5}\right)(0.055 \mathrm{~g}, 0.246 \mathrm{mmol})$ was dissolved in Milli-Q water $(18 \mathrm{ml})$ and the resulting solution, maintained at $40{ }^{\circ} \mathrm{C}$, was first partially neutralised with $\mathrm{Ba}(\mathrm{OH})_{2} \cdot 8 \mathrm{H}_{2} \mathrm{O}(0.082 \mathrm{~g}, 0.258 \mathrm{mmol})$ and subsequently treated with a solution of $\left[\mathrm{Pt}\left(\mathrm{OSO}_{3}\right)\left(\mathrm{OH}_{2}\right)(\mathrm{en})\right]$ ( $0.200 \mathrm{~g}, 0.541 \mathrm{mmol}$, in $14 \mathrm{ml}$ of Milli-Q water). The obtained suspension was kept under magnetic stirring at $40{ }^{\circ} \mathrm{C}$ overnight and then cooled down in an ice bath. The white precipitate $\left(\mathrm{BaSO}_{4}\right)$ was removed by filtration through a plug of Celite ${ }^{\circledR}$ and

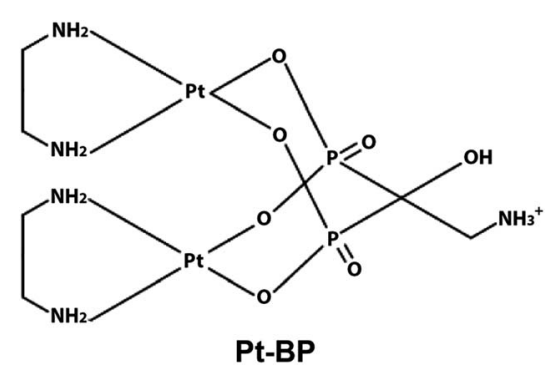

Fig. 1 Chemical structure of platinum-bisphosphonate (Pt-BP). 
the filtrate was concentrated to a volume of approximately $2 \mathrm{ml}$. The $\mathrm{pH}$ of the concentrated solution was adjusted to $\sim 1.0$ by addition of $\mathrm{H}_{2} \mathrm{SO}_{4}(1 \mathrm{M})$ and the acidic solution was treated with methanol, which induced precipitation of the desired product. The precipitate was left standing at $4{ }^{\circ} \mathrm{C}$ for $3 \mathrm{~h}$ and then isolated by filtration, washed with methanol and diethyl ether and dried under vacuum. Obtained $0.183 \mathrm{~g}$ (85\% yield). Anal. calc. for $\left[\{\mathrm{Pt}(\mathrm{en})\}_{2}\left(\mu-\mathrm{AHBP}-\mathrm{H}_{2}\right)\right]\left(\mathrm{HSO}_{4}\right) \cdot 3 \mathrm{H}_{2} \mathrm{O} \quad\left(\mathrm{C}_{6} \mathrm{H}_{29} \mathrm{~N}_{5} \mathrm{O}_{14} \mathrm{P}_{2} \mathrm{Pt}_{2} \mathrm{~S}, M_{\mathrm{w}}=\right.$ $879.4 \mathrm{~g} \mathrm{~mol}^{-1}$ ): C, 8.19\%; H, 3.32\%; N, 7.96\%. Found: C, 8.49\%; $\mathrm{H}, 3.19 \%$; N $7.64 \%$. ESI-MS: calc. for $\left[\{\mathrm{Pt}(\mathrm{en})\}_{2}\left(\mu-\mathrm{AHBP}-\mathrm{H}_{2}\right)\right]^{+}$ $\left(\left[\mathrm{C}_{6} \mathrm{H}_{22} \mathrm{~N}_{5} \mathrm{O}_{7} \mathrm{P}_{2} \mathrm{Pt}_{2}\right]^{+}\right)=728.03$; found: $m / z$ (\% relative to the base peak) $=727.8(100)[\mathrm{M}]^{+}$.

The spectroscopic characterisation of Pt-BP was consistent with literature data $\left({ }^{1} \mathrm{H}\right.$ and ${ }^{13} \mathrm{P}$ NMR spectroscopy and Fourier Transform Infrared Spectroscopy (FTIR)). Electrospray Ionisation-Mass Spectrometry (ESI-MS) was carried out using an electrospray interface and ion trap mass spectrometer (1100 Series LC/MSD Trap system Agilent, Palo Alto, CA). Elemental analyses were carried out using a Hewlett Packard 185 C, H and N analyser.

The dinuclear Pt-BP complex was found to be stable in neutral aqueous solution even after standing for several months at room temperature, as evidenced by NMR spectroscopy (data not shown).

\section{Preparation of unfunctionalised gelatine nanoparticles}

Gelatine nanoparticles (Gel) were prepared using a two-step desolvation method as previously reported. ${ }^{\mathbf{1 2 , 6 0 , 6 1}}$ Subsequently, GA was used to crosslink the nanoparticles with a molar ratio of GA relative to the amine groups in gelatine (GA/ $\mathrm{NH}_{2}$ ) of 2. After crosslinking at room temperature for $16 \mathrm{~h}$ (although 3-4 h crosslinking was sufficient to obtain stable nanoparticles, the reaction was aged for $16 \mathrm{~h}$ to obtain the highest yield), $100 \mathrm{ml}$ of glycine solution $(100 \mathrm{mM})$ was added to the gelatine nanoparticles suspension to block the unreacted aldehyde groups. After three cycles of centrifugation (5000 rpm for $60 \mathrm{~min}$, Universal $32 \mathrm{R}$ Hettich Centrifuge, Tuttlingen, Germany) and resuspension in Milli-Q water by vortexing, the $\mathrm{pH}$ of the suspensions was adjusted to 7.0. To investigate the properties of the gelatine nanoparticles in swollen state, small fractions of the suspension were stored at $4{ }^{\circ} \mathrm{C}$ until further use, whereas the rest of the nanoparticles were lyophilised (no cryoprotectant applied; Freezone 4.5, Labconco, USA) and stored at $4{ }^{\circ} \mathrm{C}$.

\section{Preparation of alendronate-functionalised gelatine nanoparticles}

ALN-functionalised gelatine nanoparticles (Gel·ALN) were prepared according to an already reported method. ${ }^{12}$ Briefly, using a two-step desolvation method, gelatine nanoparticles were prepared as previously described. ${ }^{\mathbf{1 2 , 6 0 , 6 1}}$ Subsequently, GA was used to crosslink gelatine nanoparticles with a $\mathrm{GA} / \mathrm{NH}_{2}$ molar ratio of 2 . Residual aldehyde groups from the GA crosslinking reaction were used for further conjugation with the amine $\left(\mathrm{NH}_{2}\right)$ groups of ALN. ALN was added to the gelatine nanoparticles suspension after aging the crosslinking reaction for $16 \mathrm{~h}$ (post-functionalisation, no $\mathrm{pH}$ manipulation $(\mathrm{pH} \sim 4)$ ). The conjugation reaction was carried out at room temperature with molar ratios of ALN relative to GA (ALN/GA) of 0.12, 2.5 and 5. After three cycles of centrifugation (5000 rpm for $60 \mathrm{~min}$ ) and resuspension in Milli-Q water by vortexing, the $\mathrm{pH}$ of the suspension was adjusted to 7.0. To investigate the properties of the functionalised gelatine nanoparticles in swollen state, small fractions of the suspension were stored at $4{ }^{\circ} \mathrm{C}$ until further use, whereas the rest of the nanoparticles were lyophilised and stored at $4{ }^{\circ} \mathrm{C}$.

\section{Preparation of Pt-BP-functionalised gelatine nanoparticles}

Gelatine nanoparticles were prepared using a two-step desolvation method as previously reported. ${ }^{\mathbf{1 2 , 6 0 , 6 1}}$ Subsequently, GA was used to crosslink gelatine nanoparticles with a $\mathrm{GA} / \mathrm{NH}_{2}$ molar ratio of 2 . Residual aldehyde groups from the GA crosslinking reaction were used for further conjugation with the amine $\left(\mathrm{NH}_{2}\right)$ groups of Pt-BP. Pt-BP was added to the gelatine nanoparticles suspension after aging the crosslinking reaction for $16 \mathrm{~h}$ (post-functionalisation, no $\mathrm{pH}$ manipulation $(\mathrm{pH} \sim 4)$ ). The conjugation reaction was carried out at room temperature. The efficiency of the conjugation reaction was investigated at Pt$\mathrm{BP} / \mathrm{GA}$ molar ratios of $0.06,0.12,0.24$ and 0.48 . After three cycles of centrifugation (5000 rpm for $60 \mathrm{~min}$ ) and resuspension in Milli-Q water by vortexing, the $\mathrm{pH}$ of the suspensions was adjusted to 7.0. To investigate the properties of the functionalised gelatine nanoparticles in swollen state, small fractions of the suspension were stored at $4{ }^{\circ} \mathrm{C}$ until further use, whereas the rest of the gelatine nanoparticles were lyophilised and stored at $4{ }^{\circ} \mathrm{C}$.

\section{Preparation of dual-functionalised gelatine nanoparticles}

Gelatine nanoparticles were prepared using a two-step desolvation method as reported previously. ${ }^{\mathbf{1 2 , 6 0 , 6 1}}$ Subsequently, GA was used to crosslink gelatine nanoparticles with a $\mathrm{GA} / \mathrm{NH}_{2}$ molar ratio of 2 . Residual aldehyde groups from the GA crosslinking reaction were used for further conjugation with the amine $\left(\mathrm{NH}_{2}\right)$ groups of both ALN and Pt-BP. To evaluate and optimise the dual conjugation reaction, different functionalisation schemes and molar ratios between the reactants were selected for the addition of ALN and Pt-BP to the suspension of gelatine nanoparticles (see Table 1 for an overview of the abbreviations, compositions and functionalisation parameters):

- Gelatine nanoparticles functionalised with ALN only $(\mathrm{Gel} \cdot \mathrm{ALN})$;

- Gelatine nanoparticles functionalised with Pt-BP only $(\mathrm{Gel} \cdot \mathrm{Pt})$

- Gelatine nanoparticles simultaneously functionalised with ALN and Pt-BP (Gel-ALN·Pt-Sim);

- Gelatine nanoparticles sequentially functionalised with ALN and Pt-BP $($ Gel-ALN//Pt);

- Gelatine nanoparticles sequentially functionalised with PtBP and ALN (Gel·Pt//ALN).

All the conjugation reactions were carried out at room temperature after aging the GA crosslinking reaction for $16 \mathrm{~h}$. After three cycles of centrifugation (5000 rpm for $60 \mathrm{~min}$ ) and 
Table 1 Abbreviations, compositions and functionalisation parameters of unfunctionalised and (dual-)functionalised gelatine nanoparticles

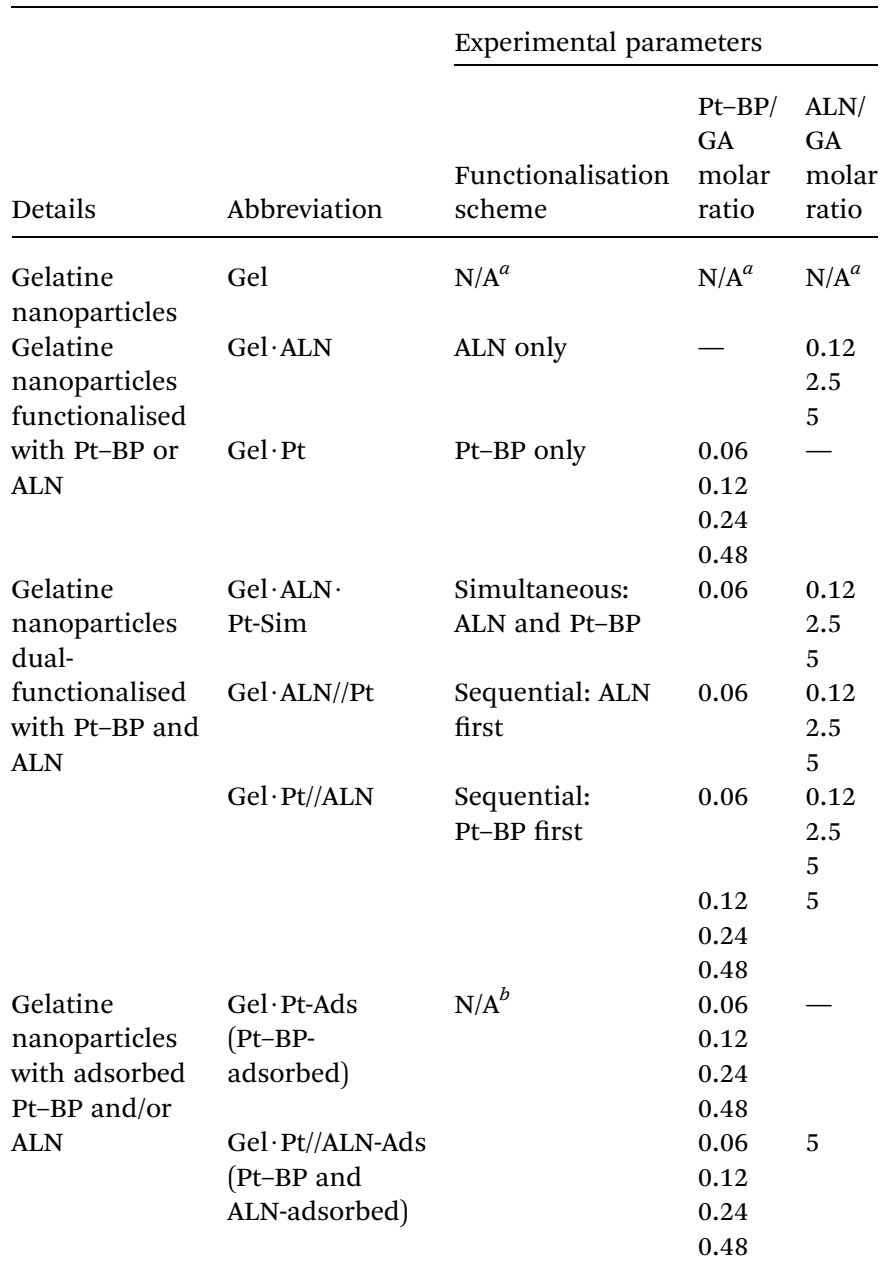

${ }^{a}$ No ALN- or Pt-BP-functionalisation carried out for Gel. ${ }^{b}$ No ALN- or Pt-BP-functionalisation, but only physical adsorption, carried out for Gel-Pt-Ads and Gel·Pt//ALN-Ads.

resuspension in Milli-Q by vortexing, the $\mathrm{pH}$ of the suspension was adjusted to 7.0 by using 1 or $0.1 \mathrm{M} \mathrm{NaOH}$ solutions. To investigate the properties of the functionalised gelatine nanoparticles in swollen state, small fractions of the suspension were stored at $4{ }^{\circ} \mathrm{C}$ until further use, whereas the rest of the nanoparticles were lyophilised and stored at $4{ }^{\circ} \mathrm{C}$.

As a control experiment, similar amounts of Pt-BP and ALN (Table 1) were physically adsorbed onto a suspension of unfunctionalised gelatine nanoparticles $\left(\mathrm{GA} / \mathrm{NH}_{2}\right.$ molar ratio = 2; crosslinked at room temperature for $16 \mathrm{~h}$; blocked with $100 \mathrm{ml}$ of $100 \mathrm{mM}$ glycine solution). After addition of Pt-BP and/ or ALN to the suspension of unfunctionalised gelatine nanoparticles, the mixture was left stirring at room temperature for $16 \mathrm{~h}$, after which the gelatine nanoparticles were centrifuged (5000 rpm for $60 \mathrm{~min}$ ) and resuspended in Milli-Q water by vortexing three times. The $\mathrm{pH}$ of the final suspension was adjusted to 7.0 by using 1 or $0.1 \mathrm{M} \mathrm{NaOH}$ solutions. To investigate the properties of the nanoparticles in swollen state, small fractions of the suspension were stored at $4{ }^{\circ} \mathrm{C}$ until further use, whereas the rest of the nanoparticles were lyophilised and stored at $4{ }^{\circ} \mathrm{C}$.

\section{Characterisation of gelatine nanoparticles}

Electron microscopy. The morphology and size of the gelatine nanoparticles were characterised in lyophilised state using Scanning Electron Microscopy (SEM, JEOL 6340F; all samples were mounted on aluminium stubs using carbon tape and sputter-coated with gold-palladium). Digital image analysis software (Image J, NIH) was applied to analyse the diameter of at least 200 nanoparticles using scanning electron micrographs in lyophilised state.

Water content. To study the influence of Pt-BP functionalisation as well as the dual functionalisation on the crosslinking efficiency of the gelatine nanoparticles, their water content was measured according to a method described previously. ${ }^{12}$ Briefly, $\sim 10 \mathrm{mg}$ of gelatine nanoparticles were soaked overnight at room temperature in $2 \mathrm{ml}$ of Milli-Q water while agitated on a shaking table at $90 \mathrm{rpm}$. The swollen nanoparticles were collected after $24 \mathrm{~h}$ on filter paper to remove surface water and placed in a vial of known weight $\left(W_{\text {vial }}\right)$. The weight of the vial including the swollen nanoparticles was then recorded ( $W_{\text {swollen+vial }}$ ). After lyophilising for $24 \mathrm{~h}$, the weight of the vial including the dry nanoparticles was recorded ( $\left.W_{\text {dry+vial }}\right)$. Accordingly, the water content (\%) of gelatine nanoparticles was calculated as follows:

$$
\begin{aligned}
\text { Water content }(\%)= & \frac{\left(W_{\text {swollen+vial }}-W_{\text {vial }}\right)-\left(W_{\text {dry }+ \text { vial }}-W_{\text {vial }}\right)}{W_{\text {swollen+vial }}-W_{\text {vial }}} \\
& \times 100 \\
= & \frac{W_{\text {swollen+vial }}-W_{\text {dry }+ \text { vial }}}{W_{\text {swollen }+ \text { vial }}-W_{\text {vial }}} \times 100
\end{aligned}
$$

This parameter provides an indication of the water uptake (wt\%) of the gelatine nanoparticles. Five samples were measured for each experimental group.

Dynamic light scattering and laser doppler velocimetry. Dynamic Light Scattering (DLS) and Laser Doppler Velocimetry (LDV) were performed on a Zetasizer® Nano-S (Malvern Instruments Ltd.) to characterise the hydrodynamic size (dispersed in Milli-Q water, solid content $0.01 \% \mathrm{w} / \mathrm{v}$ ) and $\zeta$-potential (dispersed in $5 \mathrm{mM}$ HEPES buffer at $\mathrm{pH} 7.0$ ) of the gelatine nanoparticles, respectively.

$\zeta$-Potential, size and polydispersity index (PDI) of the nanoparticles were also studied as a function of time at storage condition $\left(4{ }^{\circ} \mathrm{C}\right)$ as well as physiological temperature $\left(37{ }^{\circ} \mathrm{C}\right)$.

Elemental analysis. Inductively Coupled Plasma-Optical Emission Spectrometry (ICP-OES, iCap 6300) was used to quantify the degree of functionalisation with Pt-BP, dual functionalisation (Pt-BP and $\mathrm{ALN}$ ), as well as the retention of $\mathrm{Pt}-\mathrm{BP}$ and ALN to the gelatine nanoparticles. To quantify the degree of functionalisation, the nanoparticles were digested in concentrated nitric acid (at $37{ }^{\circ} \mathrm{C}$ overnight on a shaking 
table) and subsequently diluted to $1 \%$ nitric acid solutions for ICP-OES analysis of the platinum and phosphorus content.

Solid-state nuclear magnetic resonance (SS-NMR) spectroscopy. The conjugation reaction was investigated using ${ }^{13} \mathrm{C}$ and ${ }^{31} \mathrm{P}$ solid-state NMR spectroscopy. All ${ }^{31} \mathrm{P}$ solid-state NMR experiments were performed on a Varian NMR spectrometer operating at proton frequency of $850 \mathrm{MHz}$. Spectra were acquired using a $1.6 \mathrm{~mm}$ magic angle spinning (MAS) HXY probehead at a spinning frequency of $35 \mathrm{kHz} .{ }^{31} \mathrm{P}$ chemical shifts were referenced to the known resonance of $\mathrm{H}_{3} \mathrm{PO}_{4}(0 \mathrm{ppm})$. The temperature was stabilised at $298 \mathrm{~K}$. Single pulse excitation was used for obtaining the ${ }^{31} \mathrm{P}$ spectra; no proton decoupling was applied. The recycle delay was $60 \mathrm{~s}$ to make sure that the samples can be interpreted quantitatively. ${ }^{13} \mathrm{C}$ solid-state Cross-Polarisation Magic Angle Spinning (CPMAS) spectra were measured by means of a $600 \mathrm{MHz}$ Varian Spectrometer. Samples were packed into $3.2 \mathrm{~mm}$ rotors, employing a MAS rate of $10 \mathrm{kHz}$. A $90^{\circ}$ proton pulse of $2.5 \mu$ s excited transverse proton magnetisation, then 2 ms cross-polarisation pulses were simultaneously applied on both - proton and carbon channels - to induce magnetisation transfer. Two-pulse phase-modulated (TPPM) decoupling ${ }^{62}$ at a radiofrequency-field strength of $100 \mathrm{kHz}$ was used during acquisition. Carbon chemical shifts were referenced with respect to tetramethylsilane (TMS) using the adamantane signals as a secondary reference.

Alendronic acid (as reference) was crushed to increase the surface of ALN crystals. As control experiment, Pt-BP and/or PtBP mixed with crushed alendronic acid was mixed with unblocked and glycine-blocked unfunctionalised gelatine nanoparticles.

Energy-dispersive X-ray (EDX) spectroscopy. Energydispersive X-ray (EDX) measurements were performed using a Field Emission Scanning Electron Microscope (FE-SEM; Sigma 300, Zeiss, Germany) equipped with an EDX detector (XFlash detector 610M, Brucker Nano GmbH, Germany). Prior to the measurements, the samples were coated with a $10 \mathrm{~nm}$ thick chromium layer. Each EDX spectrum was collected for 5 min by scanning over an area of $1200 \times 900$ spots with the spot size of $0.38 \mu \mathrm{m}$. The elemental composition of each sample was determined automatically by the EDX (ESPRIT 2.0) software.

Retention and release of alendronate and Pt-BP to gelatine nanoparticles. The retention of Pt-BP and/or ALN to gelatine nanoparticles was evaluated in Milli-Q water as a function of soaking time (1, 3, 7 and 14 days). Phosphate buffered saline (PBS), cell culture medium or similar phosphorus-containing media could not be used for this experiment, since the high phosphate concentration in the media did not allow for detection and differentiation of phosphorus released from dual- or Pt-BP-functionalised gelatine nanoparticles using ICP-OES.

Briefly, $10 \mathrm{mg}$ of lyophilised functionalised gelatine nanoparticles $\left(\mathrm{GA} / \mathrm{NH}_{2}\right.$ molar ratio $\left.=2 ; \mathrm{pH}_{\text {functionalisation }}=4\right)$ was added to $10 \mathrm{ml}$ of Milli-Q water at $37{ }^{\circ} \mathrm{C}$ on a shaking table (70 $\mathrm{rpm})$. At each time point, the suspensions were centrifuged at $5000 \mathrm{rpm}$ for $60 \mathrm{~min}$. The gelatine pellet was separated from the supernatant and re-suspended in $10 \mathrm{ml}$ of fresh Milli-Q water for further time points. After the last time point, to quantify the retained Pt-BP and/or ALN, the gelatine pellet was digested in concentrated nitric acid (at $37{ }^{\circ} \mathrm{C}$ overnight on a shaking table) and subsequently diluted to $1 \%$ nitric acid solutions for ICPOES analysis of the platinum and phosphorus content. To evaluate the in vitro release of platinum and ALN from gelatine nanoparticles, the collected supernatants were also mixed with nitric acid to prepare a $1 \%$ nitric acid solution (and kept at $37^{\circ} \mathrm{C}$ overnight on a shaking table) for further elemental analysis of the platinum and phosphorus content using ICP-OES. The retention and release of $\mathrm{Pt}-\mathrm{BP}$ and/or ALN to gelatine nanoparticles with adsorbed Pt-BP (and/or ALN) was evaluated similarly as a control experiment.

\section{Affinity of gelatine nanoparticles to calcium phosphate}

Commercially available titanium discs of $12 \mathrm{~mm}$ diameter and $1.5 \mathrm{~mm}$ thickness were grit-blasted with aluminium oxide particles, cleaned ultrasonically in isopropanol ( $\sim 15 \mathrm{~min})$ and air-dried overnight. The surface roughness of the titanium discs $\left(R_{\mathrm{a}}=2.0 \pm 0.2 \mu \mathrm{m}\right)$ was measured using a universal surface tester (Innowep GmbH, Würzburg, Germany). Titanium discs were subsequently coated with a thin hydroxyapatite film $(1.5 \pm$ $0.1 \mu \mathrm{m}$ ) using a commercially available radiofrequency magnetron sputter deposition system (Edwards High Vacuum ESM 100 system, Crawford, England) as described previously. ${ }^{\mathbf{6 3} 64}$ The target materials were hydroxyapatite $\left(\mathrm{Ca}_{10}(-\right.$ $\left.\mathrm{PO}_{4}\right)_{6}(\mathrm{OH})_{2}$ ) granules (diameter: 0.5-1.0 mm; CAM Bioceramics BV, Leiden, the Netherlands). The discs were mounted on a rotating water-cooled substrate holder. The distance between target and implant holder was $80 \mathrm{~mm}$. Before sputtering, the discs were cleaned by etching for $\sim 10$ min with argon ions. During deposition $(7.5 \mathrm{~h})$, the argon pressure was kept at $5 \times$ $10^{-3}$ mbar and the sputter power of 2 times $400 \mathrm{~W}$ with a coating thickness of $\sim 1 \mu \mathrm{m}$ was used. After deposition, the coated implants were subjected to an additional infrared heat treatment for $30 \mathrm{~s}$ at $650{ }^{\circ} \mathrm{C}$ (Quad Ellipse Chamber, Model E4$10 \mathrm{P}$, Research Inc.), as previously described. ${ }^{65}$ The titanium discs were eventually cleaned ultrasonically in isopropanol for $\sim 15$ min to remove any unbound particles, air-dried and stored at room temperature. The coatings were characterised by thin film XRD (PW 1830, PANalytical) and ATR-FTIR (Spectrum One ${ }^{\mathrm{TM}}$, Perkin Elmer).

The affinity of gelatine nanoparticles to $\mathrm{CaP}$ was evaluated by immersing the hydroxyapatite-coated titanium discs for $16 \mathrm{~h}$ in $2 \mathrm{ml}$ of gelatine nanoparticle suspension (dispersed at $2.5 \mathrm{mg}$ $\mathrm{ml}^{-1}$ in $5 \mathrm{mM}$ HEPES buffer) at $37{ }^{\circ} \mathrm{C}$ on a shaking plate. The titanium discs were horizontally placed at the bottom of 24-well cell culture plates without any constraint for horizontal movement within the well. The autofluorescence of gelatine was exploited to quantify the amount of surface-bound gelatine nanoparticles by fluorescence microscopy using an automated axio Imager Z1 microscope (Carl Zeiss Micro Imaging $\mathrm{GmbH}$, Göttingen, Germany) and digital image analysis (Image J, NIH) after thoroughly rinsing the discs with Milli-Q water to remove the unbound gelatine nanoparticles. The affinity of gelatine nanoparticles to the hydroxyapatite surfaces was quantified by dividing the areas covered by gelatine nanoparticles to the entire 
hydroxyapatite-covered surface area of the titanium discs using the autofluorescence detected from gelatine nanoparticles.

\section{Statistical analysis}

All results are depicted as mean \pm standard deviation. The statistical analyses were performed using GraphPad Prism (version 5.03) software. Differences among groups were determined by one-way analysis of variance (ANOVA) with a Bonferroni (multiple comparisons) post-hoc test, and a value of $p<$ 0.05 was considered as significantly different.

\section{Results and discussion}

\section{Preparation and characterisation of Pt-BP}

The dinuclear Pt(II) complex bis-\{ethylenediamineplatinum(II)\}2-amino-1-hydroxyethane-1,1-diyl-bisphosphonate (Pt-BP, Fig. 1) was prepared according to a method previously described. ${ }^{16}$ Briefly, the barium salt of the aminobisphosphonate was first prepared and subsequently treated with two equivalents of $\left[\mathrm{Pt}\left(\mathrm{OSO}_{3}\right)\left(\mathrm{OH}_{2}\right)(\mathrm{en})\right]$ (en = ethylenediamine). The reaction mixture was left under magnetic stirring overnight at higher temperature $\left(40{ }^{\circ} \mathrm{C}\right)$ as compared to the previously reported procedure. ${ }^{9}$ The elemental analysis and the spectroscopic features were consistent with the data reported in the literature (data not shown).,

\section{Preparation and characterisation of gelatine nanoparticles}

Effect of functionalisation scheme. Gelatine nanoparticles (either functionalised or unfunctionalised) were prepared using an established two-step desolvation method ${ }^{60,61}$ resulting in the formation of spherical nanoparticles. Average diameters of the nanoparticles in lyophilised and swollen state are provided in Table 2. SEM micrographs revealed that the morphology and size of Pt-BP-functionalised or dual-functionalised gelatine nanoparticles in lyophilised state did not change upon functionalisation, as compared to the unfunctionalised ones (Gel) (Fig. 2). DLS measurements confirmed that the size of functionalised gelatine nanoparticles in swollen state did not change upon functionalisation (Table 2). Similar results were obtained for gelatine nanoparticles that were lyophilised and rehydrated/resuspended in Milli-Q water, $5 \mathrm{mM}$ HEPES buffer

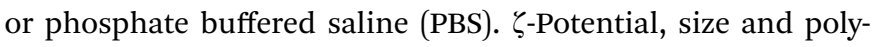
dispersity index (PDI) measurements at storage condition $\left(4^{\circ} \mathrm{C}\right)$ as well as body temperature $\left(37^{\circ} \mathrm{C}\right)$ up to 14 days showed no significant differences as compared to the results shown in Table 2.

Water content and, hence, the degree of crosslinking of either Pt-BP- or dual-functionalised gelatine nanoparticles did not change as compared to unfunctionalised gelatine nanoparticles (Table 2), as ALN and/or Pt-BP were added after completion of the crosslinking reaction and functioned mainly as blocking agents for the unreacted aldehyde groups. These results were in agreement with our previous findings for ALNfunctionalised gelatine nanoparticles. ${ }^{12}$

$\zeta$-Potential values shown in Table 2 are related to gelatine nanoparticles (dual-)functionalised at a Pt-BP/GA molar ratio of 0.06 and/or an ALN/GA molar ratio of 5. These data revealed that functionalisation of gelatine nanoparticles with negatively charged $\mathrm{ALN}^{\mathbf{1 0}}$ rendered the surface of the nanoparticles more negatively charged, irrespective of the functionalisation scheme or the Pt-BP/GA molar ratio (data not shown). The Pt-BP complex is positively charged and becomes neutral after conjugation with GA and formation of an imine bond between the pending $\mathrm{NH}_{2}$ and the aldehydes. However, functionalisation of gelatine nanoparticles with Pt-BP complex rendered the surface of the nanoparticles more negatively charged, which was similar to the $\zeta$-potential of ALN-functionalised gelatine nanoparticles. This phenomenon may not be completely explained by the experiments performed in this study. Nevertheless, we speculate that the change in $\zeta$-potential may only be the result of avoiding glycine-blocking the unreacted aldehyde groups on the surface of gelatine nanoparticles. Another possible explanation is the hydrolysis of the $\mathrm{Pt}-\mathrm{O}-\mathrm{P}$ bonds in the conjugated Pt-BP complex, that could occur at neutral $\mathrm{pH}$ with entering of one or more water molecules in the coordination sphere of platinum and formation of $\mathrm{P}-\mathrm{O}^{-}$groups. Water molecules coordinated to positively charged platinum ions are acidic and tend to deprotonate with formation of neutral Pthydroxido species. ${ }^{67,68}$ The overall result would be the presence of $\mathrm{P}-\mathrm{O}^{-}$groups on the surface of nanoparticles, which could contribute to lowering the $\zeta$-potential of the Pt-BPfunctionalised gelatine nanoparticles.

As shown in Fig. 3, ICP-OES measurements confirmed the presence of platinum in Pt-BP-functionalised gelatine nanoparticles. At the lowest $\mathrm{Pt}-\mathrm{BP} / \mathrm{GA}$ molar ratio of 0.06 , it was possible to link approximately 5.3 moles of Pt-BP to one mole of gelatine (Gel·Pt[0.06]), whereas for Pt-BP-adsorbed gelatine nanoparticles with the same $\mathrm{Pt}-\mathrm{BP} / \mathrm{GA}$ molar ratio (Gel·Pt-Ads [0.06]) only 0.6 moles of Pt-BP per mole of gelatine was

Table 2 Particle size, $\zeta$-potential and water content of unfunctionalised and (dual-)functionalised gelatine nanoparticles ${ }^{a}$

\begin{tabular}{|c|c|c|c|c|c|c|}
\hline Particle size (lyophilised, $\mathrm{nm})^{b}$ & $179 \pm 51$ & $181 \pm 48$ & $190 \pm 36$ & $187 \pm 46$ & $188 \pm 32$ & $192 \pm 40$ \\
\hline Particle size (swollen, $\mathrm{nm})^{b}$ & $395 \pm 4$ & $401 \pm 7$ & $387 \pm 11$ & $376 \pm 20$ & $394 \pm 9$ & $382 \pm 15$ \\
\hline Water content (\%) & $89.3 \pm 2.4$ & $90.2 \pm 1.8$ & $89.1 \pm 3.1$ & $91.0 \pm 2.7$ & $89.9 \pm 3.3$ & $91.3 \pm 1.6$ \\
\hline
\end{tabular}

${ }^{a}$ The presented results are related to gelatine nanoparticles with Pt-BP/GA molar ratio of 0.06 and/or ALN/GA molar ratio of $5 .{ }^{b}$ Particle sizes of lyophilised gelatine nanoparticles were measured by analysing at least 200 particles using electron microscopy, whilst the diameters of swollen nanoparticles were determined by DLS. ${ }^{a}$ Denotes statistical differences versus the other experimental groups within the same row $(p<0.05)$. 

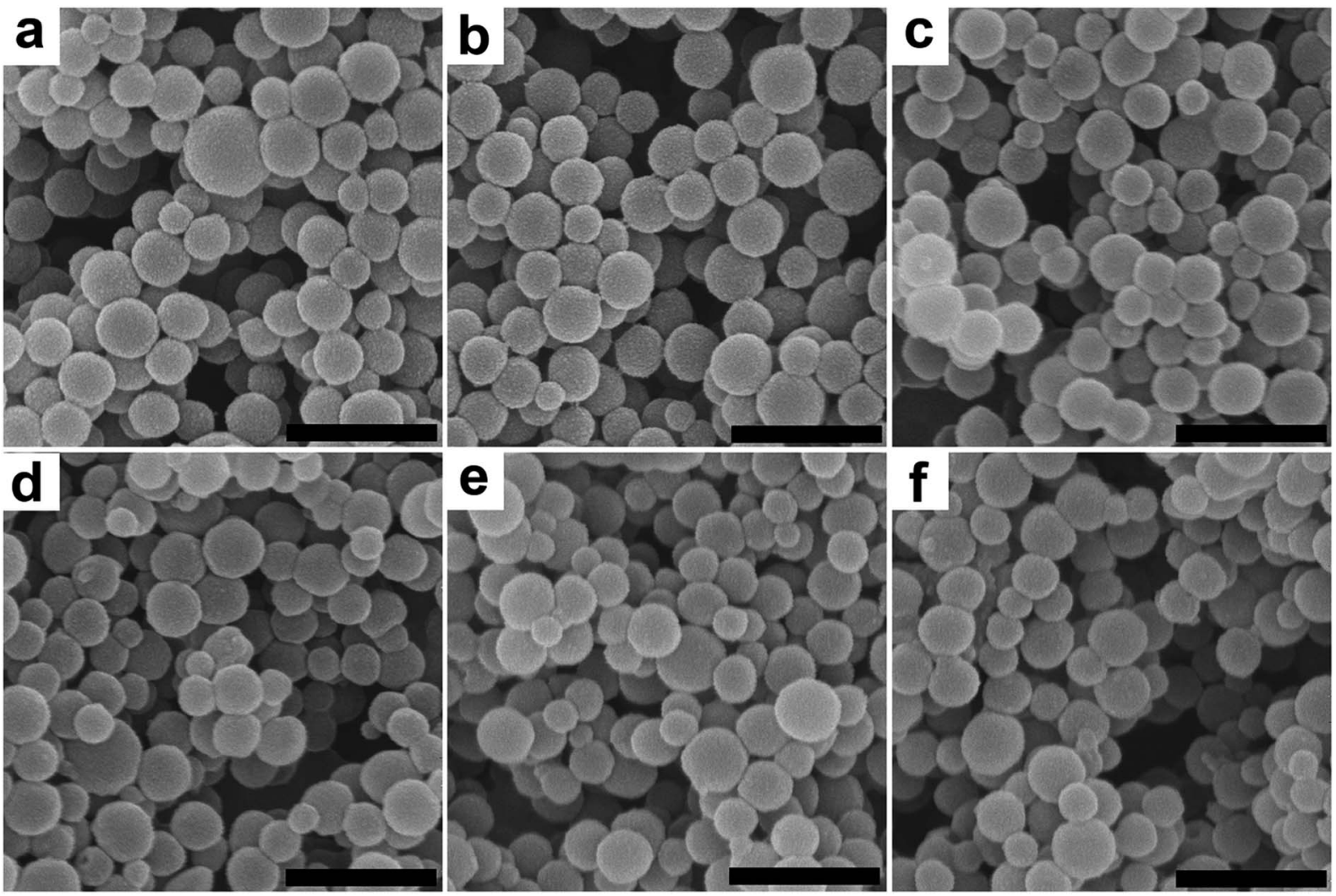

Fig. 2 Scanning electron micrographs of gelatine nanoparticles. (a) unfunctionalised (Gel); (b) ALN-functionalised (Gel·ALN); (c) Pt-BP-functionalised (Gel-Pt); (d) simultaneously functionalised with ALN and Pt-BP (Gel-ALN.Pt-Sim); (e) sequentially functionalised with Pt-BP/then/ALN $(\mathrm{Gel} \cdot \mathrm{Pt} / / \mathrm{ALN}) ;(\mathrm{f})$ sequentially functionalised with ALN/then/Pt-BP (Gel·ALN//Pt) (scale bar $=500 \mathrm{~nm})$.

detected. By increasing the Pt-BP/GA molar ratio up to 0.48 , the amount of Pt-BP linking increased up to approximately 28 moles of Pt-BP per mole of gelatine. The total amount of Pt-BP present on gelatine nanoparticles with adsorbed $\mathrm{Pt}-\mathrm{BP}$, however, was significantly lower as compared to those functionalised with $\mathrm{Pt}-\mathrm{BP}$ at all $\mathrm{Pt}-\mathrm{BP} / \mathrm{GA}$ molar ratios shown in Fig. 3.

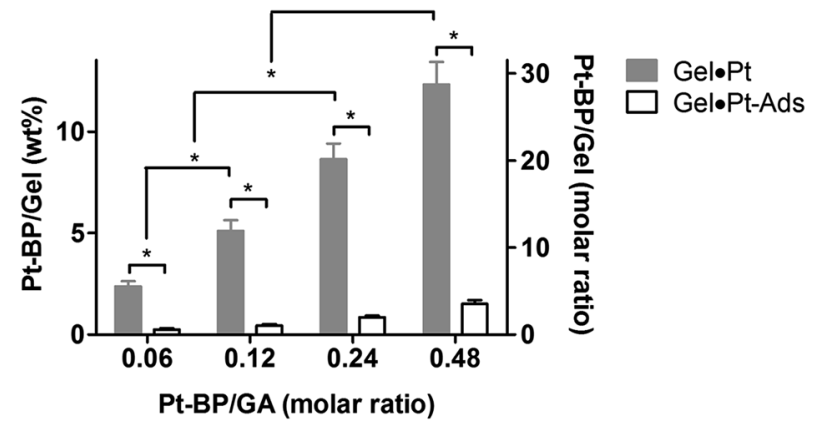

Fig. 3 Amount of platinum in gelatine nanoparticles measured by ICP$\mathrm{OES}$ (the left $Y$-axis represents relative weight percentage of $\mathrm{Pt}-\mathrm{BP}$ present in gelatine nanoparticles (wt\%), whereas the right $Y$-axis represents the molar ratio of $\mathrm{Pt}-\mathrm{BP}$ relative to gelatine) $(p<0.05)$; $(\mathrm{Gel} \cdot \mathrm{Pt}=$ gelatine nanoparticles with covalently conjugated Pt-BP; $\mathrm{Gel} \cdot \mathrm{Pt}-\mathrm{Ads}=$ gelatine nanoparticles with physically adsorbed Pt-BP).
Fig. 4 shows the weight percentages of platinum and phosphorus for all functionalisation schemes in Pt-BP- and dualfunctionalised gelatine nanoparticles (at a fixed Pt-BP/GA molar ratio of 0.06). The ALN content in samples was calculated by subtracting the amount of phosphorus derived from Pt-BP from the total amount of detected phosphorus.

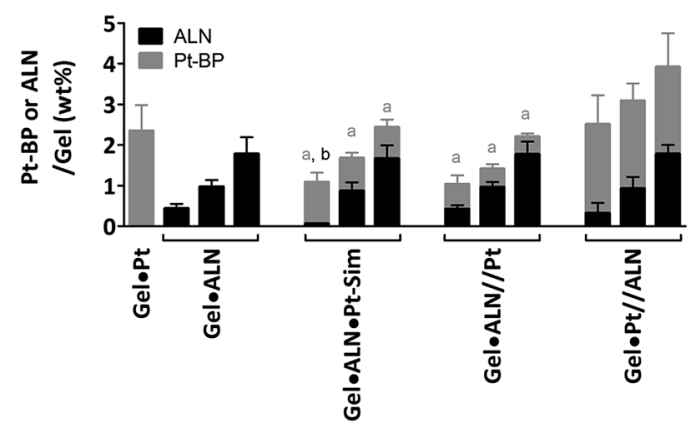

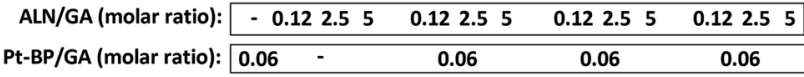

Fig. 4 Effect of functionalisation scheme and ALN/GA molar ratio on the efficiency of dual-functionalisation of gelatine nanoparticles measured by ICP-OES ( $Y$ axis represents wt\% of Pt-BP and ALN present in gelatine nanoparticles; Letters "a" and "b" denote statistically significant differences versus Gel·Pt and Gel·ALN[0.06], respectively) $(p<0.05)$. 
Corresponding molar ratios of detected Pt-BP or ALN relative to gelatine can be found in Fig. S1. $\uparrow$ As shown in Fig. 4 and S1, $\uparrow$ the efficiency of Pt-BP conjugation reaction decreased regardless of the applied ALN/GA molar ratio by simultaneous addition of ALN and Pt-BP to gelatine nanoparticles after completion of the GA crosslinking reaction. This was attributed to the competition between Pt-BP and ALN to react with available free aldehyde groups on gelatine nanoparticles. Moreover, Pt-BP molecules were less likely to link to the available free aldehyde groups on gelatine nanoparticles, since ALN/GA molar ratios were higher than $\mathrm{Pt}-\mathrm{BP} / \mathrm{GA}$ ratios.

The amount of Pt-BP linked to gelatine nanoparticles decreased considerably for all ALN/GA molar ratios (Fig. 4) when the nanoparticles were first functionalised with ALN. This is obviously due to the fact that the majority of available free aldehyde groups on gelatine nanoparticles were already consumed by the reaction with ALN. The amount of either PtBP or ALN linked to gelatine nanoparticles did not decrease when the nanoparticles were first functionalised with $\mathrm{Pt}-\mathrm{BP}$ prior to the reaction with ALN. Therefore, the latter dualfunctionalisation scheme and the highest ALN/GA molar ratio were selected for further experiments.

By increasing the Pt-BP/GA molar ratio up to 0.48 , the efficiency of the Pt-BP conjugation reaction for dual-functionalised gelatine nanoparticles increased (Fig. 5; molar ratios of detected $\mathrm{Pt}-\mathrm{BP}$ or ALN in different samples can be found in Fig. S2 $\dagger$ ), which was similar to our results for Pt-BP-functionalised gelatine nanoparticles shown in Fig. 3. However, no significant change was observed with respect to the efficiency of the ALN conjugation reaction (Fig. 5 and $\mathrm{S} 2 \dagger$ ), which indicated that the ALN-functionalisation reaction was independent from the preceding reaction with $\mathrm{Pt}-\mathrm{BP}$.

Energy-dispersive X-ray (EDX) spectroscopy confirmed the presence of platinum on dual- and Pt-BP-functionalised gelatine nanoparticles, whereas no platinum was detected on unfunctionalised and ALN-functionalised gelatine nanoparticles (Fig. S3†). The presence of phosphorus was also confirmed for gelatine nanoparticles functionalised with alendronate and Pt-BP (Fig. S3†).

Since the amount of Pt-BP attached to gelatine nanoparticles relative to the gelatine matrix itself was very low, the obtained

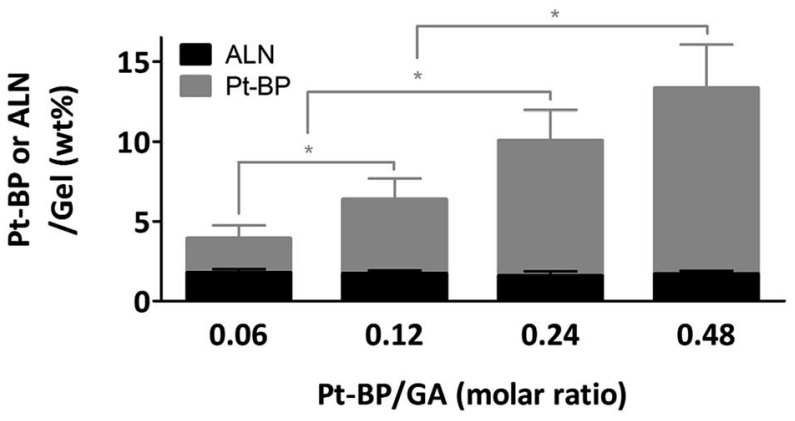

Fig. 5 Effect of Pt-BP/GA molar ratio on the efficiency of dualfunctionalisation of gelatine nanoparticles measured by ICP-OES ( $Y$ axis represents $w t \%$ of $\mathrm{Pt}-\mathrm{BP}$ and $\mathrm{ALN}$ present in gelatine nanoparticles) $(p<0.05)$. solid-state NMR spectra showed very low signal intensity from the Pt-BP complex linked to gelatine nanoparticles (Fig. S4 $\dagger$ ). Furthermore, ${ }^{13} \mathrm{C}$ spectra are dominated by the gelatine signals, which overlap all signals from other moieties. As a result, we could not observe free alendronate signals in the ${ }^{13} \mathrm{C}$ spectrum. Further investigations using higher Pt-BP concentrations are required to obtain clear and conclusive solid-state NMR spectra from (dual-)functionalised gelatine nanoparticles.

Retention of Pt-BP and alendronate to gelatine nanoparticles. To evaluate the extent and stability of the covalent linking between Pt-BP and gelatine, the retention of Pt-BP to gelatine nanoparticles was studied as a function of soaking time in Milli-Q water. Retention tests showed that at the lowest Pt$\mathrm{BP} / \mathrm{GA}$ molar ratio of 0.06 , up to $97 \mathrm{wt} \%$ of the Pt-BP initially present on Pt-BP-functionalised gelatine nanoparticles ( $\mathrm{Gel} \cdot \mathrm{Pt}$ [0.06]) was retained after 14 days of soaking (Fig. 6a). On the contrary, less retention of $\mathrm{Pt}-\mathrm{BP}$ was observed for gelatine nanoparticles containing adsorbed Pt-BP (Gel-Pt-Ads[0.06]) as compared to the functionalised ones, since $85 \mathrm{wt} \%$ of the initially bound Pt-BP was retained to Gel-Pt-Ads[0.06] after only 14 days of soaking. Similarly, higher cumulative release of platinum (\% $M / M_{\text {tot }}$ ) was observed for Pt-BP-functionalised gelatine nanoparticles when compared to the Pt-BP-adsorbed ones at the lowest Pt-BP/GA ratio of 0.06 (Fig. 6b; Gel $\cdot \mathrm{Pt}[0.06]$ versus $\mathrm{Gel} \cdot \mathrm{Pt}$-Ads[0.06]; Fig. 6c and d show statistical analysis of Fig. $6 \mathrm{a}$ and $\mathrm{b}$, respectively, for day 14 only). Up to Pt-BP/GA ratio of 0.24, Pt-BP-functionalised and Pt-BP-adsorbed gelatine nanoparticles showed a similar behaviour. Pt-BP-adsorbed gelatine nanoparticles at highest $\mathrm{Pt}-\mathrm{BP} / \mathrm{GA}$ molar ratio (Gel·Pt-Ads[0.48]), however, displayed considerable differences with respect to cumulative platinum release ( $\left.\begin{array}{ll}\% & M / M_{\text {tot }}\end{array}\right)$ compared to all the other groups (Fig. 6b and d).

When the Pt-BP/GA molar ratio was increased up to $0.12,0.24$ and 0.48, gelatine nanoparticles functionalised with Pt-BP did not show more $\mathrm{Pt}-\mathrm{BP}$ retention as compared to the corresponding gelatine nanoparticles containing adsorbed Pt-BP (Fig. 6a and c). Interestingly, even at the highest $\mathrm{Pt}-\mathrm{BP} / \mathrm{GA}$ ratio of 0.48 , more than $80 \mathrm{wt} \%$ of the Pt-BP initially present on the gelatine nanoparticles was retained to the nanoparticles after 14 days of soaking. Pt-BP generally showed strong retention (higher than 75 $\mathrm{wt} \%$ ) to both types of gelatine nanoparticles containing Pt-BP (adsorbed or functionalised) after 14 days (Fig. 6a and b). Nevertheless, the extent at which Pt-BP can be loaded onto (Fig. 3) and released from gelatine nanoparticles is limited when Pt-BP is adsorbed. For functionalised gelatine nanoparticles, on the other hand, it is possible to adjust the desired amount of released $\mathrm{Pt}-\mathrm{BP}$ by varying the $\mathrm{Pt}-\mathrm{BP} / \mathrm{GA}$ molar ratio.

Fig. 7a compares the retention of $\mathrm{Pt}-\mathrm{BP}$ to dualfunctionalised gelatine nanoparticles versus gelatine nanoparticles functionalised with Pt-BP only. The results revealed that dual-functionalisation did not have a considerable influence on the retention efficiency of Pt-BP to gelatine nanoparticles as compared to those functionalised with $\mathrm{Pt}-\mathrm{BP}$ only. Furthermore, Pt-BP generally showed strong retention (higher than $80 \mathrm{wt} \%$ ) to dual-functionalised gelatine nanoparticles. Cumulative platinum release $\left(\begin{array}{ll}\% & M / M_{\text {tot }}\end{array}\right)$ from dualfunctionalised gelatine nanoparticles increased by increasing 
a)

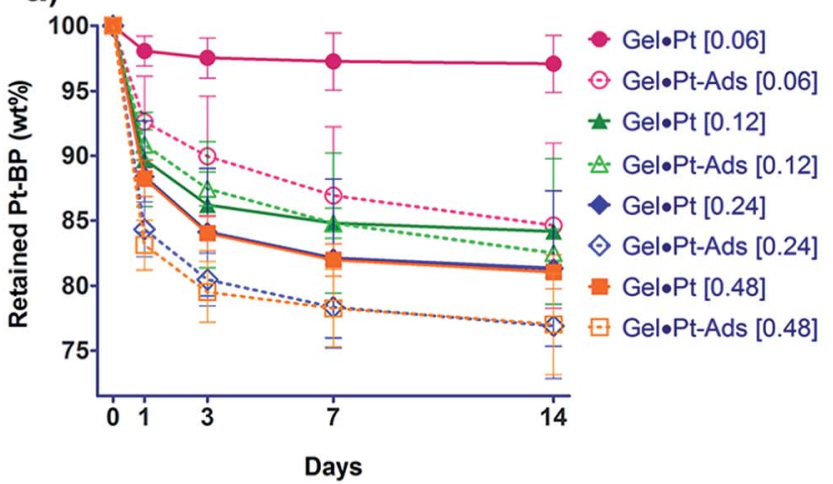

b)
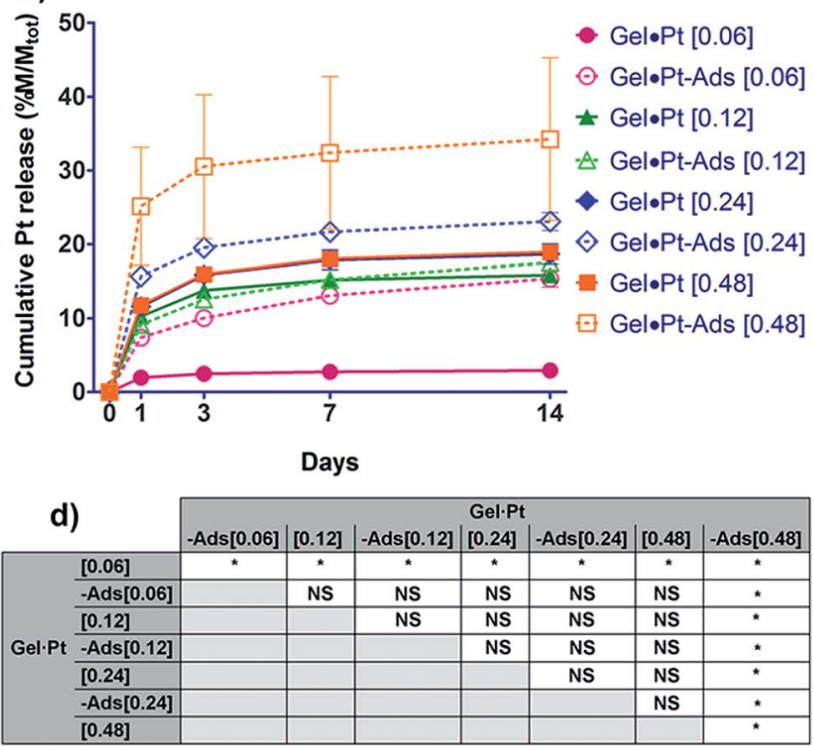

Fig. 6 (a) Weight percentage of Pt-BP retained to Pt-BP-functionalised versus Pt-BP-adsorbed gelatine nanoparticles as a function of soaking time and Pt-BP/GA molar ratio; (b) cumulative release of total amount of platinum relative to platinum initially detected on 10 mg of gelatine nanoparticles (\% $M / M_{\text {tot }}$ ) as a function of soaking time and Pt-BP/GA molar ratio; (c) and (d) statistical analysis of (a) and (b), respectively, for day 14 only (*: $p<0.05$; NS: not statistically different).

the Pt-BP/GA molar ratio, which was similar to gelatine nanoparticles functionalised with Pt-BP only (Fig. 7b-d show statistical analysis of Fig. 7a and b, respectively, for day 14 only).

Similar to the results obtained for Pt-BP-adsorbed and PtBP-functionalised gelatine nanoparticles (Fig. 6b), dualfunctionalised gelatine nanoparticles displayed increased cumulative platinum release (Fig. S5b and $d \dagger$ ) for all Pt-BP/GA molar ratios, when compared to those with adsorbed $\mathrm{Pt}-\mathrm{BP}$ and
ALN. For dual-functionalised gelatine nanoparticles, however, it was possible to detect differences in Pt-BP retention, when compared to those with adsorbed Pt-BP and ALN (Fig. S5a and $\left.\mathrm{c}^{\dagger}\right)$. Nevertheless, it should be emphasised that even gelatine nanoparticles adsorbed with Pt-BP and ALN displayed high PtBP retention properties after 14 days (approximately higher than $65 \%$ for the group with the highest Pt-BP/GA molar ratio of $0.48(\mathrm{Gel} \cdot \mathrm{Pt} \cdot \mathrm{ALN}-\mathrm{Ads}[0.48]))$.

\section{a)}

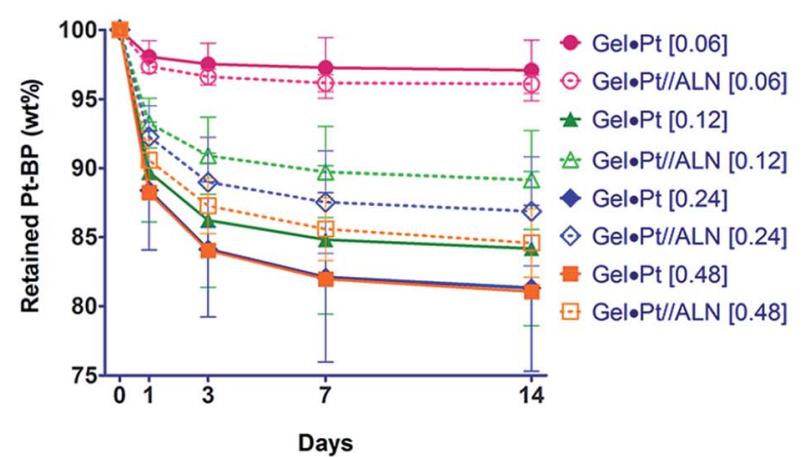

\section{c)}

\begin{tabular}{|c|c|c|c|c|c|c|c|}
\hline 1 & //ALN[0.06] & [0.12] & | I/ALN[0.12] & {$[0.24]$} & | IIALN[0.24] & {$[0.48]$} & | IIALN[0.48] \\
\hline$[0.06]$ & NS & $\star$ & * & $*$ & $\star$ & $*$ & $\star$ \\
\hline IIALN[0.06] & & * & NS & * & * & * & * \\
\hline$[0.12]$ & & & NS & NS & NS & NS & NS \\
\hline Gel-Pt /IALN[0.12] & & & & * & NS & $*$ & NS \\
\hline$[0.24]$ & & & & & NS & NS & NS \\
\hline IIALN[0.24] & & & & & & NS & NS \\
\hline$[0.48]$ & & & & & & & NS \\
\hline
\end{tabular}

b)
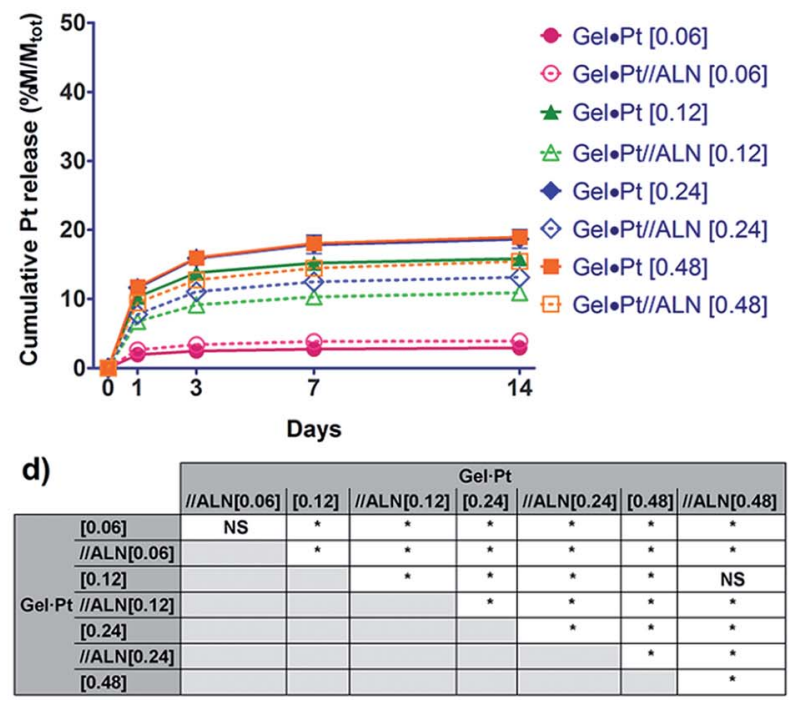

Fig. 7 (a) Weight percentage of Pt-BP retained to dual-functionalised versus Pt-BP-functionalised gelatine nanoparticles as a function of soaking time and Pt-BP/GA molar ratio; (b) cumulative release of total amount of platinum relative to platinum initially detected on 10 mg of gelatine nanoparticles (\% M/M tot $)$ as a function of soaking time and Pt-BP/GA molar ratio; (c) and (d) statistical analysis of (a) and (b), respectively, for day 14 only (*: $p<0.05$; NS: not statistically different). 
a)

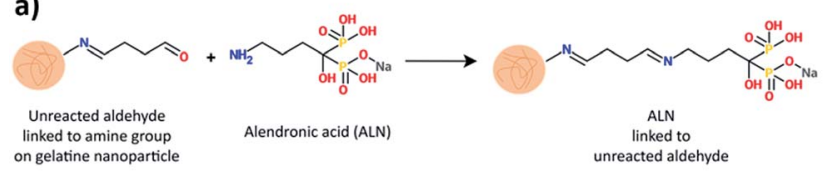

b)

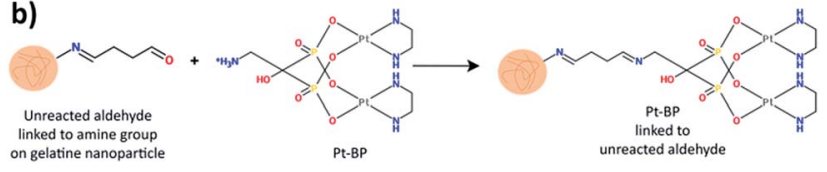

Fig. 8 Potential schemes for the reaction between (a) ALN and (b) PtBP complex with aldehyde residues on gelatine nanoparticles.

The Pt-BP conjugation reaction resulted into significantly improved retention of $\mathrm{Pt}-\mathrm{BP}$ to dual-functionalised gelatine nanoparticles as compared to nanoparticles which were loaded with Pt-BP and ALN by physical adsorption only. The increased amount of Pt-BP linked to chemically functionalised gelatine nanoparticles (Fig. 3) and the improved retention of Pt-BP on dual-functionalised gelatine nanoparticles (Fig. S5†) was attributed to the formation of covalent imine linkages between aldehyde groups at the nanoparticle surface and the pending amine group of the Pt-BP complex. Fig. 8 shows potential schemes for the reactions between ALN (Fig. 8a) and the Pt-BP complex (Fig. 8b) with aldehyde residues as present on gelatine nanoparticles. The hydrolytic stability of this bond was more than two weeks, which would be sufficient for the intended applications. Moreover, in the tumour microenvironment, where protease such as matrix metalloproteinases (collagenase and gelatinase) are highly expressed, the proteolytic degradation of the gelatine nanoparticles could possibly occur at a faster rate than in the blood with the simultaneous increased release of the Pt-BP complex. ${ }^{69,70}$ Several studies from our group confirmed that release of loaded compounds from gelatine nanoparticles is controlled by proteolytic enzymes when sufficiently strong bonds are formed between the loaded compounds and gelatin carriers. ${ }^{71-73}$ The results presented in the current study showed that Pt-BP complex displays a considerable retention to gelatine nanoparticles; therefore, it can be assumed that the release of linked Pt-BP from these (dual-)functionalized nanoparticles will also be controlled to a certain extent by proteolytic enzymes. This, however, should be investigated in more details, e.g. by using radioactive ${ }^{195 \mathrm{~m}} \mathrm{Pt}$.

The fast release of $\sim 15-20 \mathrm{wt} \%$ of platinum from (dual-) functionalised gelatine nanoparticles at $0.12,0.24$ and 0.48 Pt-BP/GA molar ratios (Fig. 6, 7 and S5 $\dagger$ ) was attributed to physically entrapped Pt-BP rather than cleavage of imine bonds. This phenomenon was in line with the broadly reported burst release of various biomolecules adsorbed onto or encapsulated inside gelatine nanoparticles without formation of
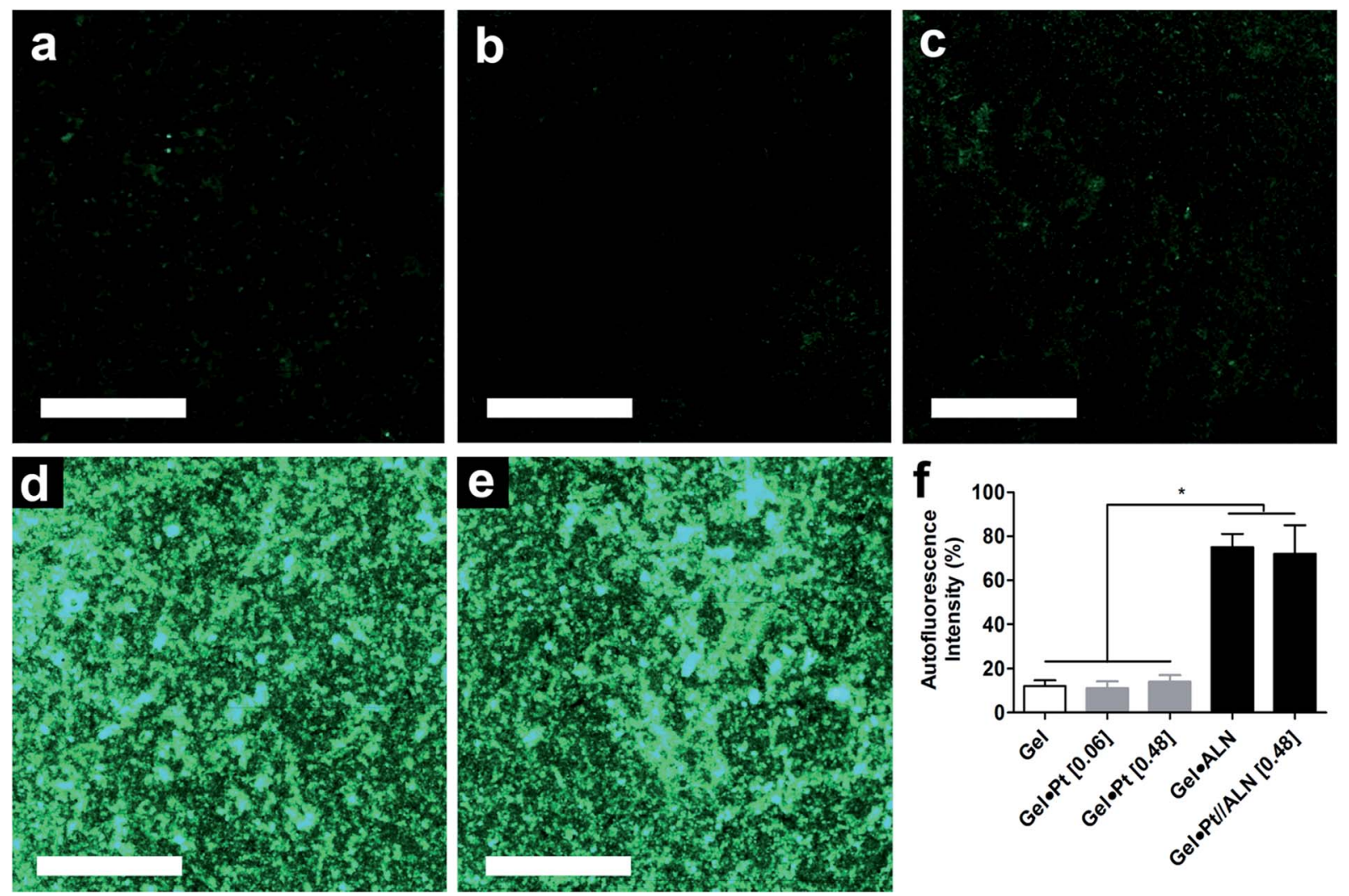

Fig. 9 Fluorescence images of hydroxyapatite-coated titanium discs immersed in suspensions of (a) Gel, (b) Gel·Pt[0.06], (c) Gel·Pt[0.48], (d) $\mathrm{Gel} \cdot \mathrm{ALN}$ and (e) Gel.Pt//ALN[0.48] (scale bar $=1 \mathrm{~mm}$ ). (f) Quantification of the fluorescence intensity derived from the autofluorescence of surface-bound gelatine nanoparticles $(p<0.05)$. 
covalent bonds between the biomolecules and the polymeric macromers. ${ }^{12,45,74-76}$

By comparing the ALN retention of dual-functionalised gelatine nanoparticles with ALN-functionalised gelatine nanoparticles (Fig. S6†), ${ }^{12}$ we observed that dual-functionalisation decreased the retention of ALN to gelatine nanoparticles from approximately $70 \mathrm{wt} \%$ down to approximately $50 \mathrm{wt} \%$. Increasing the Pt-BP/GA molar ratio, however, did not affect the retention of ALN of the dual-functionalised gelatine nanoparticles. These findings suggest that the affinity of mineralbinding ALN-functionalised gelatine nanoparticles to calcium phosphate (CaP) could be maintained even after dualfunctionalisation with high amounts of Pt-BP.

Affinity of gelatine nanoparticles to calcium phosphate. To investigate the effect of Pt-BP-functionalisation as well as dualfunctionalisation on the affinity of unfunctionalised and ALNfunctionalised gelatine nanoparticles to CaP surfaces, hydroxyapatite-coated titanium discs (corresponding XRD and ATR-FTIR spectra are reported in Fig. S7a and $b, \dagger$ respectively) were immersed in gelatine nanoparticle suspensions. Pure titanium discs, hydroxyapatite-coated titanium discs, ALN and $\mathrm{Pt}-\mathrm{BP}$ did not generate any autofluorescence. Consequently, the detected fluorescence could be only attributed to the autofluorescence of gelatine nanoparticles. Digital image analysis of fluorescence micrographs of hydroxyapatite-coated titanium discs revealed that Pt-BP-functionalisation (Fig. 9a-c) did not increase the affinity of gelatine nanoparticles to hydroxyapatite surfaces (Fig. 9f). This finding is not surprising, since all the oxygen atoms of the bisphosphonate are bound to platinum. ALN- (Gel·ALN) or dual-functionalisation (Gel·Pt//ALN[0.48]) of gelatine nanoparticles (Fig. 9d and e, respectively), however, resulted into approximately sixfold higher fluorescence intensity on the hydroxyapatite surfaces compared to unfunctionalised or Pt-BP-functionalised gelatine nanoparticles. Gelatine nanoparticles dual-functionalised with lower Pt-BP/GA molar ratios $(0.06,0.12$ and 0.24$)$ also showed similar fluorescence intensities on the hydroxyapatite surfaces as shown in Fig. 9e for Pt-BP/GA molar ratio of 0.48 (data not shown). These results demonstrate that mineral-binding gelatine nanoparticles functionalised with Pt-BP molecules did not lose their mineralbinding capacity.

\section{Conclusions}

This study presents a conjugation strategy for the preparation of gelatine nanoparticles functionalised with a chemotherapeutic platinum-bisphosphonate (Pt-BP) complex or dualfunctionalised with $\mathrm{Pt}-\mathrm{BP}$ and mineral-binding alendronate (ALN). The degree of functionalisation could be tuned by varying the molar ratio of $\mathrm{Pt}-\mathrm{BP}$ and ALN relative to glutaraldehyde used as crosslinker. Sustained and tuneable release of platinum was observed from both Pt-BP- and dualfunctionalised gelatine nanoparticles. Moreover, dual- and ALN-functionalised gelatine nanoparticles displayed a high affinity to calcium phosphate as a result of functionalisation with ALN. Summarising, it can be concluded that mineralbinding gelatine nanoparticles can be functionalised with chemotherapeutic drugs, which may benefit the development of bone-seeking and potentially chemotherapeutically active carriers for treatment of bone tumours.

\section{Acknowledgements}

The authors are grateful for the funding support from NIRM, the Netherlands Institute for Regenerative Medicine and AgentschapNL (IOP Self Healing Materials, Project no. SHM012014). Tatiana Zinckevich would like to acknowledge the support from funding TA-COAST programme. The Netherlands Organisation for Scientific Research (NWO) is acknowledged for support of the solid-state NMR facility for advanced materials science. Furthermore, the authors would like to thank Dr Vincent M. Cuijpers and Martijn Martens for their practical assistance.

\section{References}

1 K. W. Hagberg, A. Taylor, R. K. Hernandez and S. Jick, Cancer Epidemiol., 2013, 37, 240-246.

2 I. G. Finlay, M. D. Mason and M. Shelley, Lancet Oncol., 2005, 6, 392-400.

3 M. R. Smith, F. Saad, R. Coleman, N. Shore, K. Fizazi, B. Tombal, K. Miller, P. Sieber, L. Karsh, R. Damiao, T. L. Tammela, B. Egerdie, H. Van Poppel, J. Chin, J. Morote, F. Gomez-Veiga, T. Borkowski, Z. Ye, A. Kupic, R. Dansey and C. Goessl, Lancet, 2012, 379, 39-46.

4 J. R. Gralow, J. S. Biermann, A. Farooki, M. N. Fornier, R. F. Gagel, R. N. Kumar, C. L. Shapiro, A. Shields, M. R. Smith, S. Srinivas and C. H. Van Poznak, J. Natl. Compr. Cancer Network, 2009, 7(suppl. 3), S1-S35.

5 A. Muvaffak, I. Gurhan, U. Gunduz and N. Hasirci, J. Drug Targeting, 2005, 13, 151-159.

6 C. L. Tseng, W. Y. Su, K. C. Yen, K. C. Yang and F. H. Lin, Biomaterials, 2009, 30, 3476-3485.

7 N. J. Wheate, S. Walker, G. E. Craig and R. Oun, Dalton Trans., 2010, 39, 8113-8127.

8 T. C. Johnstone, K. Suntharalingam and S. J. Lippard, Chem. Rev., 2016, 116, 3436-3486.

9 N. Margiotta, R. Ostuni, D. Teoli, M. Morpurgo, N. Realdon, B. Palazzo and G. Natile, Dalton Trans., 2007, 3131-3139.

10 B. Palazzo, M. Iafisco, M. Laforgia, N. Margiotta, G. Natile, C. L. Bianchi, D. Walsh, S. Mann and N. Roveri, Adv. Funct. Mater., 2007, 17, 2180-2188.

11 M. Iafisco, B. Palazzo, M. Marchetti, N. Margiotta, R. Ostuni, G. Natile, M. Morpurgo, V. Gandin, C. Marzano and N. Roveri, J. Mater. Chem., 2009, 19, 8385-8392.

12 K. Farbod, M. Diba, T. Zinkevich, S. Schmidt, M. J. Harrington, A. P. M. Kentgens and S. C. G. Leeuwenburgh, Macromol. Biosci., 2016, 16, 717-729. 13 K. Farbod, M. R. Nejadnik, J. A. Jansen and S. C. G. Leeuwenburgh, Tissue Eng., Part B, 2014, 20, 173-188. 14 S. E. Papapoulos, Bone, 2006, 38, 613-616.

15 L. E. Cole, T. Vargo-Gogola and R. K. Roeder, Adv. Drug Delivery Rev., 2015, 12-27. 
16 K. Farbod, K. Sariibrahimoglu, A. Curci, A. Hayrapetyan, J. N. W. Hakvoort, J. J. J. P. van den Beucken, M. Iafisco, N. Margiotta and S. C. G. Leeuwenburgh, Tissue Eng., Part A, 2016, 22, 788-800.

17 K. Park, Quant. Imag. Med. Surg., 2012, 2, 106-113.

18 A. O. Elzoghby, J. Controlled Release, 2013, 172, 1075-1091.

19 Z. Lu, T. K. Yeh, M. Tsai, J. L. Au and M. G. Wientjes, Clin. Cancer Res., 2004, 10, 7677-7684.

20 M. A. Lopez-Heredia, G. J. Kamphuis, P. C. Thüne, F. C. Öner, J. A. Jansen and X. F. Walboomers, Biomaterials, 2011, 32, 5411-5416.

21 H. Wang, S. C. G. Leeuwenburgh, Y. Li and J. A. Jansen, Tissue Eng., Part B, 2011, 18, 24-39.

22 D. Ding, Z. Zhu, Q. Liu, J. Wang, Y. Hu, X. Jiang and B. Liu, Eur. J. Pharm. Biopharm., 2011, 79, 142-149.

23 A. Ethirajan, U. Ziener, A. Chuvilin, U. Kaiser, H. Colfen and K. Landfester, Adv. Funct. Mater., 2008, 18, 2221-2227.

24 N. Doi, J. I. Jo and Y. Tabata, J. Biomater. Sci., Polym. Ed., 2012, 23, 991-1004.

25 M. Santoro, A. M. Tatara and A. G. Mikos, J. Controlled Release, 2014, 190, 210-218.

26 Y. Ikada and Y. Tabata, Adv. Drug Delivery Rev., 1998, 31, 287301.

27 S. Young, M. Wong, Y. Tabata and A. G. Mikos, J. Controlled Release, 2005, 109, 256-274.

28 H. Wang, Q. Zou, O. C. Boerman, A. W. Nijhuis, J. A. Jansen, Y. Li and S. C. Leeuwenburgh, J. Controlled Release, 2013, 166, 172-181.

29 J. P. Chen, Y. L. Leu, C. L. Fang, C. H. Chen and J. Y. Fang, J. Pharm. Sci., 2011, 100, 655-666.

30 F. A. Dorkoosh, M. P. Stokkel, D. Blok, G. Borchard, M. Rafiee-Tehrani, J. C. Verhoef and H. E. Junginger, J. Controlled Release, 2004, 99, 199-206.

31 J. Liu, D. Meisner, E. Kwong, X. Y. Wu and M. R. Johnston, Biomaterials, 2007, 28, 3236-3244.

32 J. Liu, D. Meisner, E. Kwong, X. Y. Wu and M. R. Johnston, Cancer Res., 2009, 69, 1174-1181.

33 S. M. Selkirk, S. J. Greenberg, R. J. Plunkett, T. A. Barone, A. Lis and P. O. Spence, Gene Ther., 2002, 9, 432-443.

34 D. Y. Sze, J. Vasc. Intervent. Radiol., 2006, 17, 163-167.

35 C. Lawlor, M. P. O'Sullivan, N. Sivadas, S. O'Leary, P. J. Gallagher, J. Keane and S. A. Cryan, Mol. Pharm., 2011, 8, 1100-1112.

36 A. S. Mundada and B. K. Shrikhande, Drug Dev. Ind. Pharm., 2006, 32, 443-448.

37 M. Rajan, V. Raj, A. A. Al-Arfaj and A. M. Murugan, Int. J. Pharm., 2013, 453, 514-522.

38 M. Saravanan, K. Bhaskar, G. Maharajan and K. S. Pillai, Int. J. Pharm., 2004, 283, 71-82.

39 Y. Fan, W. Shan-Guang, P. Yu-Fang, S. Feng-Lan and L. Tao, Drug Dev. Ind. Pharm., 2009, 35, 630-645.

40 C. Goldenstedt, A. Birer, D. Cathignol, S. Chesnais, Z. El Bahri, C. Massard, J. L. Taverdet and C. Lafon, Ultrason. Sonochem., 2008, 15, 808-814.

41 M. Saravanan, J. Anbu, G. Maharajan and K. S. Pillai, J. Drug Targeting, 2008, 16, 366-378.
42 D. Thakor, I. Spigelman, Y. Tabata and I. Nishimura, Mol. Ther., 2007, 15, 2124-2131.

43 C. Bourquin, D. Anz, K. Zwiorek, A. L. Lanz, S. Fuchs, S. Weigel, C. Wurzenberger, P. von der Borch, M. Golic, S. Moder, G. Winter, C. Coester and S. Endres, J. Immunol., 2008, 181, 2990-2998.

44 M. Brzoska, K. Langer, C. Coester, S. Loitsch, T. O. Wagner and C. Mallinckrodt, Biochem. Biophys. Res. Commun., 2004, 318, 562-570.

45 A. Kaur, S. Jain and A. K. Tiwary, Acta Pharm., 2008, 58, 6174.

46 S. J. Lee, J. Y. Yhee, S. H. Kim, I. C. Kwon and K. Kim, J. Controlled Release, 2013, 172, 358-366.

47 D. Wu and M. Wan, J. Pharm. Pharm. Sci., 2008, 11, 32-45.

48 S. Ohta, N. Nitta, A. Sonoda, A. Seko, T. Tanaka, M. Takahashi, S. Takemura, Y. Tabata and K. Murata, Eur. J. Radiol., 2009, 72, 534-540.

49 S. Gunji, K. Obama, M. Matsui, Y. Tabata and Y. Sakai, Surgery, 2013, 154, 991-999.

50 A. K. Gupta, M. Gupta, S. J. Yarwood and A. S. Curtis, J. Controlled Release, 2004, 95, 197-207.

51 D. A. Heller, Y. Levi, J. M. Pelet, J. C. Doloff, J. Wallas, G. W. Pratt, S. Jiang, G. Sahay, A. Schroeder, J. E. Schroeder, Y. Chyan, C. Zurenko, W. Querbes, M. Manzano, D. S. Kohane, R. Langer and D. G. Anderson, Adv. Mater., 2013, 25, 1449-1454.

52 N. Dixit, K. Vaibhav, R. S. Pandey, U. K. Jain, O. P. Katare, A. Katyal and J. Madan, Biomed. Pharmacother., 2015, 69, $1-10$.

53 Y. W. Won, S. M. Yoon, C. H. Sonn, K. M. Lee and Y. H. Kim, ACS Nano, 2011, 5, 3839-3848.

54 E. Leo, R. Arletti, F. Forni and R. Cameroni, Farmaco, 1997, 52, 385-388.

55 G. Y. Lee, K. Park, J. H. Nam, S. Y. Kim and Y. Byun, J. Drug Targeting, 2006, 14, 707-716.

56 K. Karthikeyan, P. Gunasekaran, N. Ramamurthy and S. Govindasamy, Pharm. Biol., 1999, 37, 285-290.

57 J. Madan, N. Dhiman, S. Sardana, R. Aneja, R. Chandra and A. Katyal, Anticancer Drugs, 2011, 22, 543-555.

58 W. A. Bubnis and C. M. Ofner, Anal. Biochem., 1992, 207, 129-133.

59 S. Piccinonna, N. Margiotta, C. Pacifico, A. Lopalco, N. Denora, S. Fedi, M. Corsini and G. Natile, Dalton Trans., 2012, 41, 9689-9699.

60 H. Wang, M. B. Hansen, D. W. Lowik, J. C. van Hest, Y. Li, J. A. Jansen and S. C. G. Leeuwenburgh, Adv. Mater., 2011, 23, H119-H124.

61 H. Wang, M. Bongio, K. Farbod, A. W. G. Nijhuis, J. J. J. P. van den Beucken, O. C. Boerman, J. C. van Hest, Y. Li, J. A. Jansen and S. C. G. Leeuwenburgh, Acta Biomater., 2014, 10, 508-519.

62 A. E. Bennett, C. M. Rienstra, M. Auger, K. V. Lakshmi and R. G. Griffin, J. Chem. Phys., 1995, 103, 6951-6958.

63 J. G. Wolke, J. P. van der Waerden, H. G. Schaeken and J. A. Jansen, Biomaterials, 2003, 24, 2623-2629. 
64 H. S. Alghamdi, R. Bosco, J. J. J. P. van den Beucken, X. F. Walboomers and J. A. Jansen, Biomaterials, 2013, 34, 3747-3757.

65 M. Yoshinari, T. Hayakawa, J. G. Wolke, K. Nemoto and J. A. Jansen, J. Biomed. Mater. Res., 1997, 37, 60-67.

66 N. Margiotta, R. Ostuni, V. Gandin, C. Marzano, S. Piccinonna and G. Natile, Dalton Trans., 2009, 1090410913.

67 M. El-Khateeb, T. G. Appleton, B. G. Charles and L. R. Gahan, J. Pharm. Sci., 1999, 88, 319-326.

68 N. Summa, W. Schiessl, R. Puchta, N. van Eikema Hommes and R. van Eldik, Inorg. Chem., 2006, 45, 2948-2959.

69 E. I. Deryugina, M. A. Bourdon, R. A. Reisfeld and A. Strongin, Cancer Res., 1998, 58, 3743-3750.

70 C. Wong, T. Stylianopoulos, J. Cui, J. Martin, V. P. Chauhan, W. Jiang, Z. Popović, R. K. Jain, M. G. Bawendi and
D. Fukumura, Proc. Natl. Acad. Sci. U. S. A., 2011, 108, 2426-2431.

71 S. Young, M. Wong, Y. Tabata and A. G. Mikos, J. Controlled Release, 2005, 109, 256-274.

72 H. A. Wang, O. C. Boerman, K. Sariibrahimoglu, Y. B. Li, J. A. Jansen and S. C. G. Leeuwenburgh, Biomaterials, 2012, 33, 8695-8703.

73 J. K. Song, J. C. E. Odekerken, D. W. P. M. Lowik, P. M. Lopez-Perez, T. J. M. Welting, F. Yang, J. A. Jansen and S. C. G. Leeuwenburgh, Macromol. Biosci., 2015, 15, 901-911.

74 A. K. Bajpai and J. Choubey, J. Mater. Sci.: Mater. Med., 2006, 17, 345-358.

75 M. G. Cascone, L. Lazzeri, C. Carmignani and Z. Zhu, J. Mater. Sci.: Mater. Med., 2002, 13, 523-526.

76 J. K. Li, N. Wang and X. S. Wu, J. Microencapsulation, 1998, 15, 163-172. 